\title{
Dynamics of Bright Solitary-waves in a General Fifth-order Shallow Water-wave Model
}

\author{
Woo-Pyo Hong \\ Department of Physics, Catholic University of Daegu, \\ Hayang, Kyongsan, Kyungbuk 712-702, South Korea \\ Reprint requests to W.-P. H.; E-mail: wphong@cuth.cataegu.ac.kr
}

Z. Naturforsch. 59a, 257 - 265 (2004); received January 15, 2004

\begin{abstract}
New analytic sech ${ }^{2}$-type traveling solitary-wave solutions, satisfying zero background at infinity, of a general fifth-order shallow water-wave model are found and compared with previously obtained non-zero background solutions. The allowed coefficient regions for the solitary-wave solutions are classified by requiring the wave number and angular frequency to be real. Detailed numerical simulations are performed to demonstrate the stability of the solitary-waves and to show the soliton-like behavior of two interacting solitary-waves. For a large nonlinear term we show the formation of a bounded state of two solitary-waves, called bion, which travels as a single coherent structure. PACS numbers: 03.40.Kf, 02.30.Jr, 47.20.Ky, 52.35.Mw
\end{abstract}

Key words: Fifth-order Shallow Water Wave Model; Analytic Solitary-wave Solution; Numerical Simulation; Stability; Interaction.

\section{Introduction}

While it is easy to write down in closed from a solitary wave solution for the simplest standard model, namely the Korteweg-de Vries (KdV) equation, it has proved quite difficult to obtain such solutions for problems from which the $\mathrm{KdV}$ equation was derived as a first approximation [1]. As such KdV hierarchy models, we investigate generalized fifth-order shallow water-wave equations, which describe certain physically-interesting (1+1)-dimensional waves [1] (see Kichenassamy and Olver [2] regarding physics motivations for this type of models):

$$
\begin{aligned}
v_{t} & +\alpha v_{x x x x x}+\mu v_{x x x}+\gamma \partial_{x}\left[2 v v_{x x}+v_{x}^{2}\right] \\
& +2 q v v_{x}+3 r v^{2} v_{x}=0
\end{aligned}
$$

where $\alpha, \mu, \gamma, q, r$ are model parameters, $v(x, t)$ is a real scalar function, and the subscripts denote partial derivatives. The equation reduces to the $\mathrm{KdV}$ equation for $\mu=1, q=3$, and $\alpha=\gamma=r=0$. Equation (1) includes some higher-order approximations to the (thirdorder) $\mathrm{KdV}$ equation, and in fact it reduces to the fifthorder KdV equation only for $q \gamma=3 r \mu$ and $3 \gamma \mu=5 q \alpha$, in which case there is a family of exact $\operatorname{sech}^{2}$-type solutions [2]. It has been shown by Benjamin et al. [3] and Weinstein [4] that solitary-wave solutions exist in the case of $\gamma=0$ and $\mu<0$. Meanwhile, Amick and Toland [5] have proved the existence of solitary-waves of speed equal to -1 , if $\alpha=1, r=\gamma=0, q=1$, and $\mu$ is smaller than some positive numbers. Kichenassamy [1] also has shown that there exist non-trivial solitary-waves if $\alpha>0, r \leq 0, q \neq 0$, and $\mu>0$, without presenting explicit analytic solitary wave solutions. More recently, some new analytic solitary-wave solutions have been found in $[10,11]$ by using the tanhmethod [6] or applying a truncated Painlevé expansion [7-9]. More recently, Coffey [12] has found two new rational solutions in the long-wave limit using the Painlevé expansion, and also pointed out the errors in the kink-type solitary-wave solutions obtained in [10].

In this paper we present new analytic sech $^{2}$ type traveling solitary-wave solutions with zero background, i.e., the wave amplitude vanishing at infinity, under some constraints among the coefficients, and study their dynamics by numerical simulations. In Sect. 2, we summarize the previously obtained analytic solitary-wave solutions [10-12]. We then find new sech ${ }^{2}$-type solitary-wave solutions with zero background and their allowed coefficient regions by requiring the wave number and angular frequency to be real. In Sect. 3, we study the dynamics of the solitary-waves with numerical simulations. In particular, we discuss the stability of the solitary-wave solution and the inter- 
action between the two solitary-waves in the presence of a strong nonlinear terms. Our conclusions follow in Section 4.

\section{Analytic Methods for Solitary-wave Solutions}

It has been shown in [10] that there exists a kinktype analytic solitary-wave solution of (1). More recently, Coffey [12] has found that an additional constraint is required for the solution and corrected a typographical error in [10]. In the following, however, we will show that another constraint is necessary for the kink-type solution to be physically meaningful. The usual ansatz for kink-type solutions with nonzero background $[6,10]$ can be set as

$$
v(x, t)=\sum_{j=0}^{N} A_{j}(t) \cdot \tanh ^{j}[k x+\omega t],
$$

where $N$ is the integer determined via the balance between the highest linear and nonlinear terms in (1), i.e., $N=2$. After some algebraic steps, the kink-type solitary-wave solution can be given as

$$
\begin{aligned}
v(x, t)= & \frac{6 \gamma k^{2}}{r}[2 \tanh [k x+\omega(k) t] \\
& \left.-\operatorname{cgsn}(\mathrm{k}) \tanh ^{2}[k x+\omega(k) t]\right] \\
& -\frac{38 \gamma^{2} k^{2}+r \mu}{2 r \gamma},
\end{aligned}
$$

where $\operatorname{csgn}=1$ for $\operatorname{Re}(x)>0$ and $\operatorname{cgsn}=-1$ for $\operatorname{Re}(x)<0$, and the angular frequency is

$$
\omega(k)=\frac{3 k}{4 \gamma^{2} r}\left(\mu^{2} r^{2}+100 \mu \gamma^{2} k^{2}+2308 \gamma^{4} k^{4}\right)
$$

under the following two constraints $[10,12]$

$$
\alpha=\frac{\gamma^{2}}{2 r}
$$

and

$$
q=\frac{2\left(r \mu+50 \gamma^{2} k^{2}\right)}{2 \gamma},
$$

from which we find the wave number as

$$
k=\frac{\sqrt{2(\gamma q-r \mu)}}{10|\gamma|} .
$$

In order to have a physically meaningful solitary-wave solution, $k$ is required to be real, resulting in an additional constraint as

$$
|\gamma q-r \mu|>0
$$

which has not been pointed out in the previous works $[10,12]$. This is an example of a traveling solitary-wave solution which does not vanish at infinity. However, this is unphysical because infinite energy can occur when integrated over $x$. A simple way to remedy this is to impose an additional constraint, i. e., $38 \gamma^{2} k^{2}+r \mu=0$, which also brings out more constraint.

We now turn to $\operatorname{sech}^{2}$-type traveling solitary-wave solutions of (1). Analytic solitary-wave solutions of $\mathrm{sech}^{2}$-type have been obtained in [11] by the truncated Painlevé expansion in the form

$$
\begin{aligned}
v(x, t) & =\phi^{-2}(x, t) \sum_{l=0}^{N=2} v_{l}(x, t) \phi^{l}(x, t) \\
& =\frac{v_{0}(x, t)}{\phi^{2}(x, t)}+\frac{v_{1}(x, t)}{\phi(x, t)}+v_{2}(x, t),
\end{aligned}
$$

where $\phi(x, t)$ is a non-singular ansatz function. The lengthy expressions for the bright and dark solitarywave solutions under some constraints can be found in [11]. Using a similar procedure as in [11], Coffey has obtained $\operatorname{sech}^{2}$-type analytic bright and dark solitary-wave solutions with nonzero background, which differ from those in [11], and has shown that in the long-wavelength limit they reduce to a rational solutions [12].

The main purpose of this section is to show that analytic solitary-wave solution of order $N=2$ with zero background can be found using a direct ansatz method in the form

$$
v(x, t)=A \operatorname{sech}^{2}[k x+\omega(k) t] .
$$

Substituting (10) in (1), expanding it to powers of tanh, and equating the coefficient of each $\tanh$-term to zero, we get the amplitude

$$
A=\frac{\left(8 \gamma \pm 2 \sqrt{16 \gamma^{2}-30 r \alpha}\right) k^{2}}{r}
$$

and the wave number

$$
\begin{aligned}
k=2 & {\left[15 \alpha\left(2 \gamma^{2}-5 r \alpha\right)(3 \mu \gamma \sqrt{R}+15 \mu r \alpha\right.} \\
& \left.\left.-12 \mu \gamma^{2}+10 q \gamma \alpha-5 q \sqrt{R} \alpha\right)\right]^{1 / 2} \\
& \cdot\left(300 \alpha^{2} r-120 \gamma^{2} \alpha\right)^{-1}
\end{aligned}
$$

where

$$
R=16 \gamma^{2}-30 r \alpha
$$


(a) $\alpha=0.1, \gamma=0.8, r=3.4133$

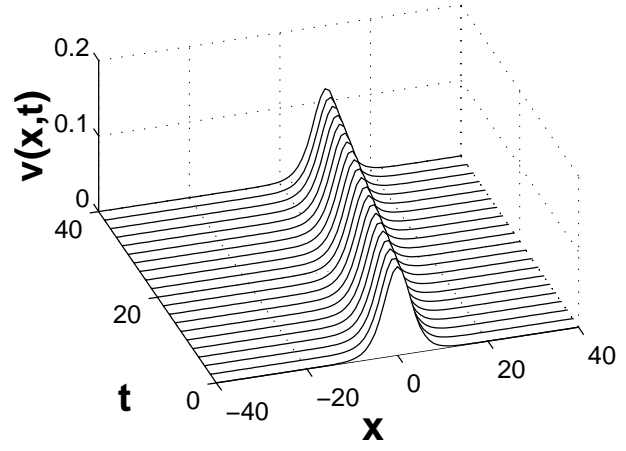

(c) $\alpha=-0.1, \gamma=-0.6, r=-1.92$

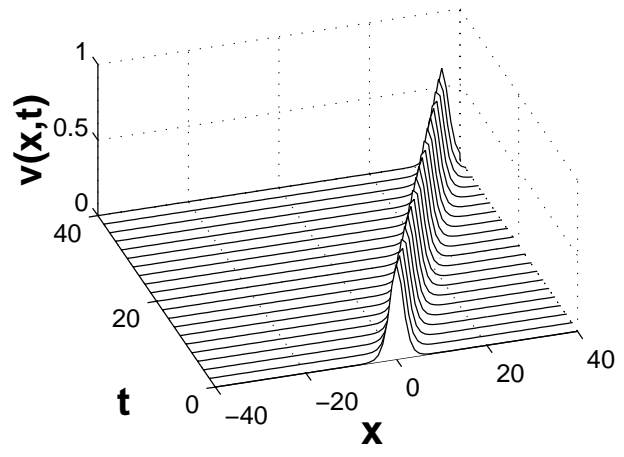

(b) $\alpha=1, \gamma=-1, r=0.53333$
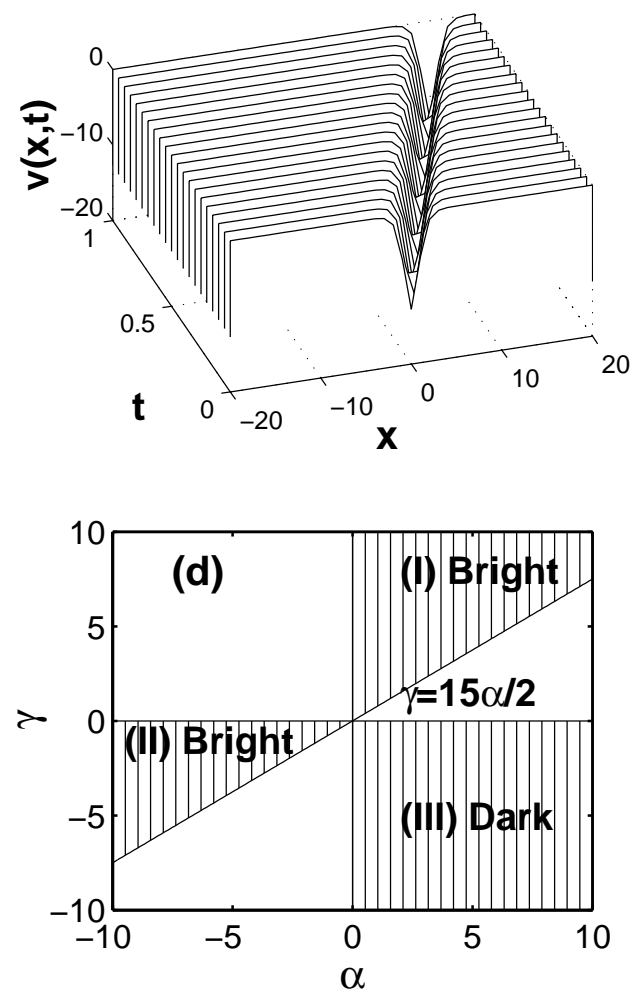

Fig. 1. (a) Bright solitary-wave solution for $\alpha>0$ and $\gamma>15 \alpha / 2$ with $r=8 \gamma^{2} / 15 \alpha$. (b) Dark solitary-wave for $\alpha>0$ and $\gamma<0$ with $r=8 \gamma^{2} / 15 \alpha$. (c) Bright solitary-wave for $\alpha<0$ and $15 \alpha / 2<\gamma<0$ with $r=8 \gamma^{2} / 15 \alpha$. (d) The allowed three coefficient regions for the solitary-waves in (16).

and the angular frequency

$$
\omega(k)=\left(24 k^{2} A \gamma-136 k^{4} \alpha-3 A^{2} r-2 A q+8 k^{2} \mu\right) k .
$$

We note that, although the solution is obtained without any constraint between the coefficients, in order to get a real wave number in (12), it is required to $R \geq 0$. In the following analysis, as an example, we assume $R=0$ to find a constraint

$$
r=\frac{8}{15} \frac{\gamma^{2}}{\alpha}
$$

which in fact indicates a balance between the highest nonlinear term with the first and second highest dispersion terms. By substituting this constraint to (11)(14), we find a new solitary-wave solution in the form

$$
v(x, t)=\frac{3(2 \mu \gamma-5 q \alpha)}{4 \gamma^{2}} \operatorname{sech}^{2}\left[k^{\prime} x+\omega^{\prime}(k) t\right],
$$

$$
\begin{aligned}
k^{\prime}= & \frac{\sqrt{5 \alpha \gamma(2 \mu \gamma-5 q \alpha)}}{10 \gamma \alpha} \\
\omega^{\prime}(k)= & \sqrt{5 \alpha \gamma(2 \mu \gamma-5 q \alpha)} \\
& \cdot\left(-14 \mu^{2} \gamma^{2}+45 \mu \gamma q \alpha-25 q^{2}\right) / 250 \gamma^{3} \alpha^{2} \\
= & \frac{k^{\prime}}{25 \gamma^{2} \alpha}\left(-14 \mu^{2} \gamma^{2}+45 \mu \gamma q \alpha-25 q^{2}\right) .
\end{aligned}
$$

By further requiring $k^{\prime}$ and $\omega^{\prime}(k)$ to be real, we find two constraints:

$$
\alpha \gamma>0 \text { and }(2 \mu \gamma-5 q \alpha)>0
$$

for a bright solitary-wave solution, and

$$
\alpha \gamma<0 \text { and }(2 \mu \gamma-5 q \alpha)<0
$$

for a dark solitary-wave solution. As usual, the group speed $v_{g}=\partial \omega^{\prime} / \partial k^{\prime}$ is given as

$$
v_{g}=\frac{(2 \mu \gamma-5 q \alpha)(5 q \alpha-7 \mu \gamma)}{25 \gamma^{2} \alpha} .
$$



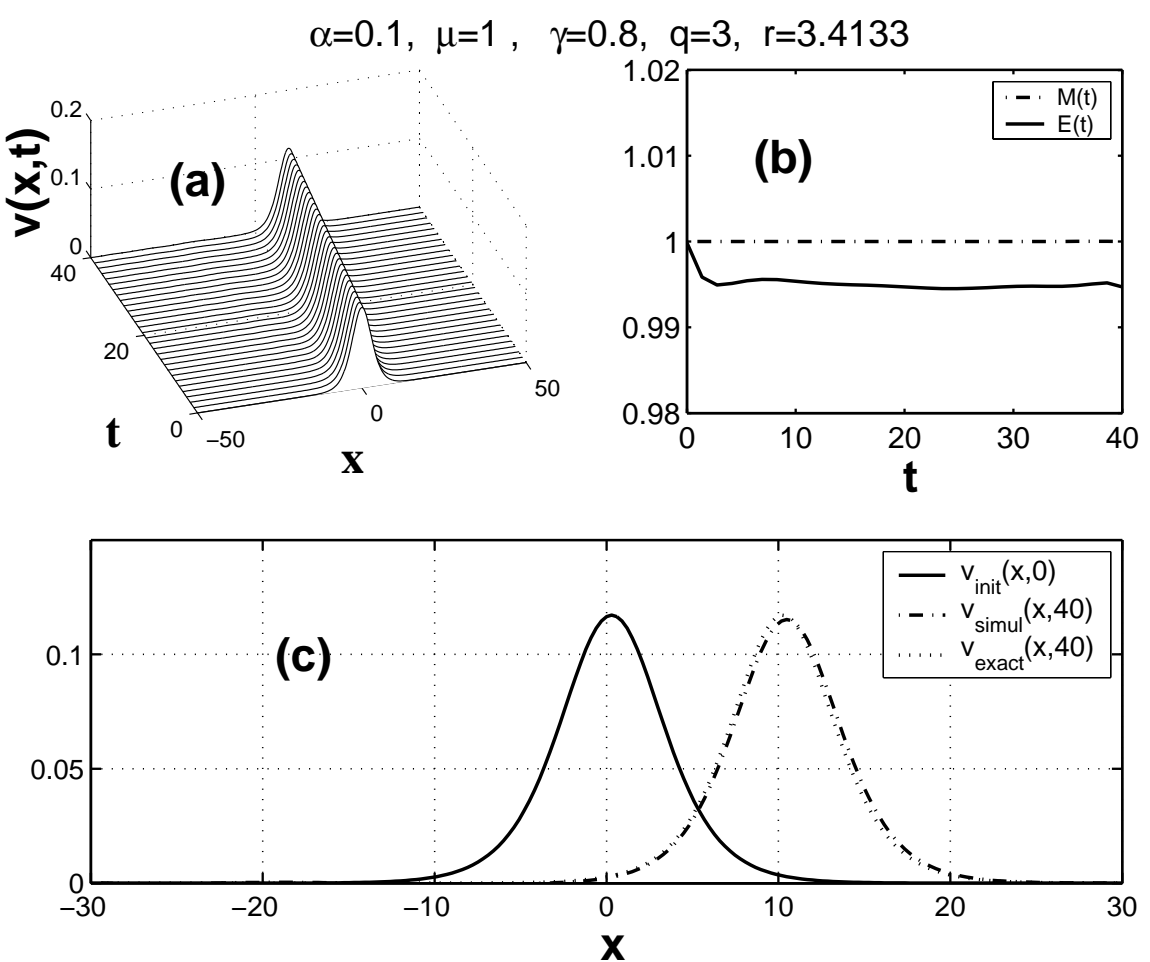

Fig. 2. (a) Evolution of the numerically simulated $v(x, t)$. (b) The less than $0.5 \%$ deviation of the normalized mass $M(t)$ and energy $E(t)$ from the initial values indicates the stability of the solitary-wave. (c) Comparison of the exact (dotted curve) with the simulated (dash-dotted curve) wave at $t=40$, using the analytic solitary-wave solution (solid curve) as the initial profile, confirms the accuracy of numerical simulation.

There are two fixed coefficients, i.e., $\mu=1$ and $q=3$, since at the $\alpha=\gamma=r=0$ limit (1) should restore to the KdV equation. It is note worthy that in a different normalization scheme one may set $\mu=1$ and $q=1$ as for the $\mathrm{KdV}$ equation. In the rest of the paper, however, we adopt $\mu=1$ and $\gamma=3$. The meaning of (15) is that $r$ is to be fixed, by fixing $\alpha$ and $\gamma$, and the solitarywave solution is given in terms of only two free coefficients: $\alpha$ and $\gamma$. From (17) $-(18)$, we find the following four possible cases: (i) $\alpha>0, \gamma>0$, and $\gamma>15 \alpha / 2$; (ii) $\alpha<0, \gamma<0$, and $\gamma>15 \alpha / 2$; (iii) $\alpha<0, \gamma>0$, and $\gamma<15 \alpha / 2$; (iv) $\alpha>0, \gamma<0$, and $\gamma<15 \alpha / 2$. Note that for case of (iii), while $\alpha<0$, both $\gamma>0$ and $\gamma<15 \alpha / 2$ are to be satisfied, which indicates that we can not obtain both real values of $k^{\prime}$ and $\omega^{\prime}$. For a classification purpose, we plot bright and dark solitary-waves in Figs. 1a and b, respectively, for the coefficients belonging to the cases of (i) and (iv). It is interesting to note that the bright solitary-wave solution also exists for case (ii), as shown in Fig. 1c, for a negative nonlinear term, i.e., $r<0$. The allowed coefficient regions for both bright and dark solitary-waves are presented in Fig. 1d. They differ from the previously obtained $q \gamma=3 r \mu$ and $3 \gamma \mu=5 q \alpha$ [2] constraints, for which (1) reduces to the KdV equation. Finally we emphasize that for $R>0$ there exist other families of $\operatorname{sech}^{2}$-type solutions under more complicated constraints between the coefficients.

\section{Numerical Study of Solitary-waves}

In this section we numerically integrate (1) to understand the stability and dynamics of the sech ${ }^{2}$-type solitary-wave solutions discussed in Section 2. Here the definition of "stability" means that the analytic solitary-wave preserves, when it is substituted in (1) and numerically integrated, its initial profile for a long propagation time without losing the total energy by radiation. The numerical scheme used in the work is based on the time advance using the Runge-Kutta fourth order scheme and a pseudospectral method using the discrete fast Fourier transformation in the spa- 

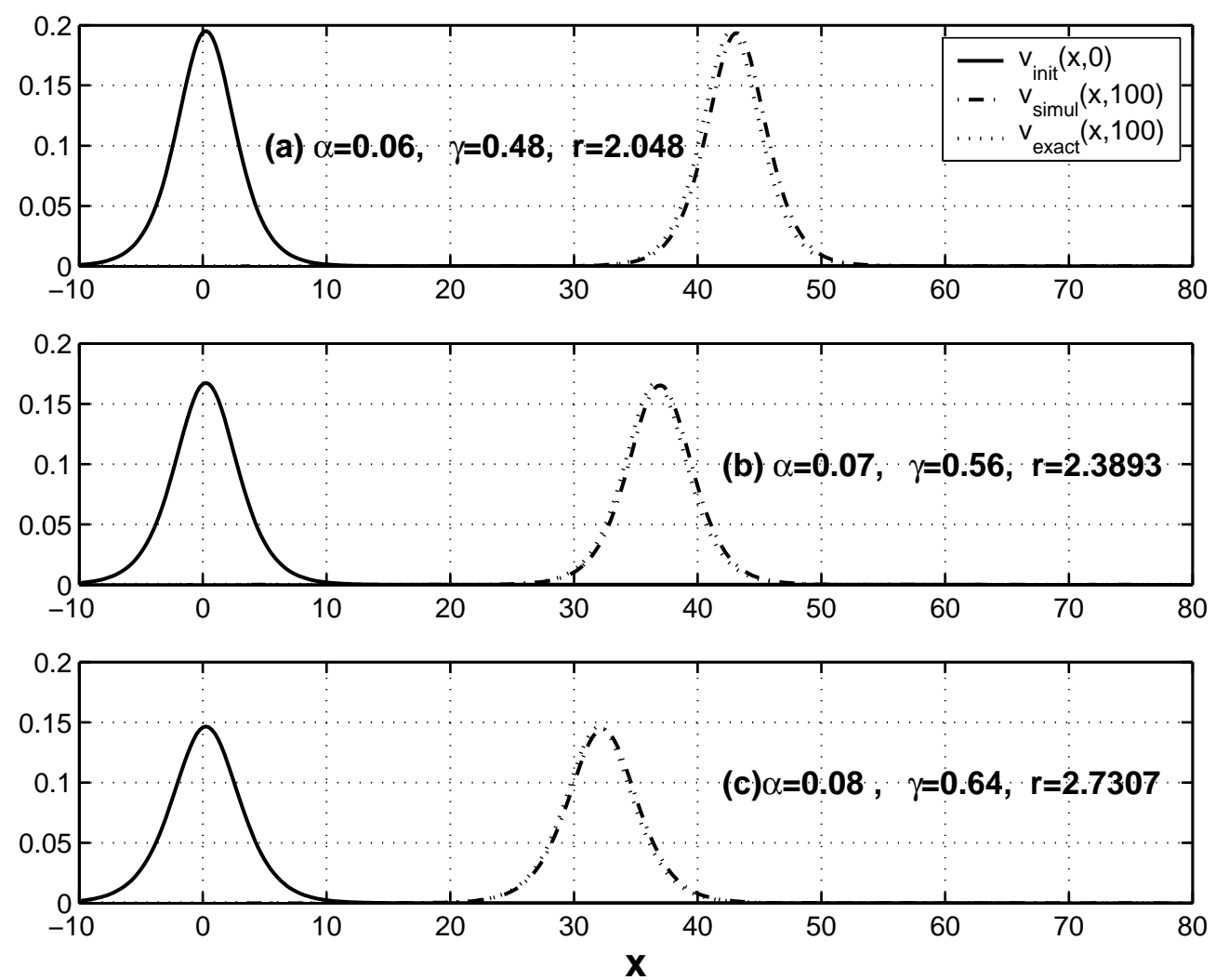

Fig. 3. Comparisons of the exact (dotted curve) vs. with the simulated (dash-dotted curve) solitary-wave at $t=100$ for three different input profiles (solid curves), which belong to the coefficient region (I) in Figure 1d. Remarkable agreements confirm the stability of the solitary-waves.

tial discretization [13], applying the periodic boundary conditions. The numerical errors in the spatial discretization were controlled by varying the number of discrete Fourier modes between 128 and 1024, and time steps between $10^{-4}$ and $10^{-2}$ are chosen for a stable wave propagation.

For the following analysis, we take the analytic bright solitary-wave from (16) as an initial profile

$$
\begin{aligned}
v_{\text {init }}(x, 0)= & \frac{3(2 \mu \gamma-5 q \alpha)}{4 \gamma^{2}} \\
& \cdot \operatorname{sech}^{2}\left[\frac{\sqrt{5 \alpha \gamma(2 \mu \gamma-5 q \alpha)}}{10 \gamma \alpha} x\right] .
\end{aligned}
$$

Using the same set of coefficients as in Fig. 1a, we show the evolution of the bright solitary-wave which preserves its initial shape and stability in Figure 2a. We note that (1) is not integrable, even though exact solitary-wave solutions exist under the constraints in (15) and (17) - (18). Therefore (1) does not possess an infinite number of time-independent integrals. However, as at least the solitary-wave solutions do exist, we want to define the simplest two such integrals, namely, the normalized mass and energy as

$$
M(t)=\int_{-\infty}^{\infty} v(x, t) \mathrm{d} x / \int_{-\infty}^{\infty} v(x, 0) \mathrm{d} x
$$

and

$$
E(t)=\int_{-\infty}^{\infty} v(x, t)^{2} \mathrm{~d} x / \int_{-\infty}^{\infty} v(x, 0)^{2} \mathrm{~d} x
$$

respectively, to control the accuracy of the numerical simulation. From Fig. 2b, we can conclude that the solitary-wave is stable as both $M(t)$ and $E(t)$ are conserved, i.e., within $0.5 \%$ deviation from the initial value, which in fact has been checked to maintain up to $t=1000$. The accuracy of the numerical scheme in this work is also clearly demonstrated in Fig. 2c by comparing the exact analytic solution $v_{\text {exact }}(x, 40)$ 

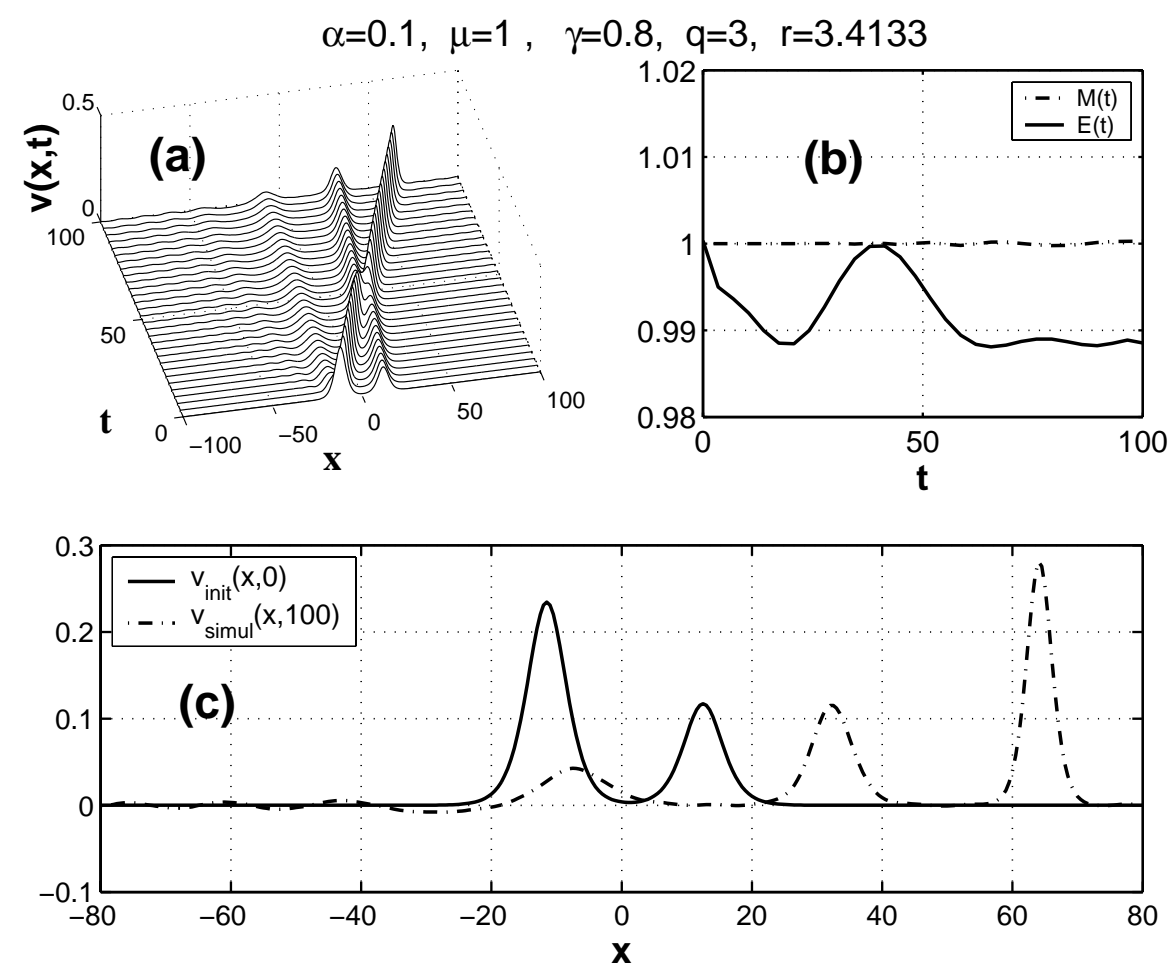

Fig. 4. (a) Evolution of the initial two solitary-waves interaction with amplitudes $B_{0}=2 A_{0}, A_{0}=0.117$, and the separation between the waves as $\delta=3$. The higher amplitude wave decays and subsequently interacts with the other wave on the right. (b) Almost elastic interaction is observed in the sense that the total energy deviates less than by $1 \%$ from the initial value and is conserved at the moment of interaction occuring $t \approx 40$. (c) The initial vs. the propagated profiles at $t=100$. Notice the appearances of three solitary-waves and the small oscillatory tail on the leftmost wave (dash-dotted curve).

(dash-dotted curve) and the group velocity $v_{g}^{\text {exact }}=$ 0.256 from (19), with $v_{\text {simul }}(x, 40)$ (dotted curve) and $v_{g}^{\text {simul }}=0.254$, respectively. The robust stability of the bright solitary-wave for a different set of coefficients, which belongs to the region (I) in Fig. 1d, is also shown in Fig. 3 by comparing the analytic solitary-wave solutions with the simulated profiles at $t=100$. Furthermore, we have numerically checked the stability of dark and bright solitary-wave solutions, of which the coefficients belong to the regions (II) and (III) in Fig. 1d, respectively, showing the same behavior as in Figs. 2-3. Therefore, in the following analysis we focus on the dynamics of bright solitary-waves of which the coefficients belong to the region (I).

The fact that (1) is nonintegrable implies that even if we can find solitary-wave solutions for particular coefficients, it is not clear whether the equation has analytic $\mathrm{N}$-soliton solutions. A question we would like to address in the present work is whether one can see from the analysis of the two waves interaction if the solitary- waves exhibit soliton-like behavior. For this purpose we consider two initial solitary-waves using the same coefficients as in Fig. 3 with initial profiles in the form

$$
v(x, 0)=A_{0} \operatorname{sech}^{2}\left(k^{\prime} x-\delta\right)+B_{0} \operatorname{sech}^{2}\left(k^{\prime} x+\delta\right),
$$

where $B_{0}=2 A_{0}$ with $A_{0}=0.117$, and the separation between the two wave is $\delta=3$. Figure 4a shows the evolution of the two solitary-waves interaction. Since the initial amplitude $B_{0}$ is twice that of the exact solution, it decomposes into the leftmost solitarywave (smaller) with an oscillatory tail, and one with higher amplitude with narrower width compared with the initial profile as shown in Fig. 4c (dash-dotted curve), respectively. Subsequently, the higher amplitude solitary-wave travels faster and interacts with the wave at the rightmost. From Fig. $4 \mathrm{~b}$ we observe that the whole interaction process is 'almost elastic' in the sense that the energy loss is less than $1 \%$ from the initial value, and it saturates to a constant value for $t>40$ owing to the presence of a small oscillatory tail. The 

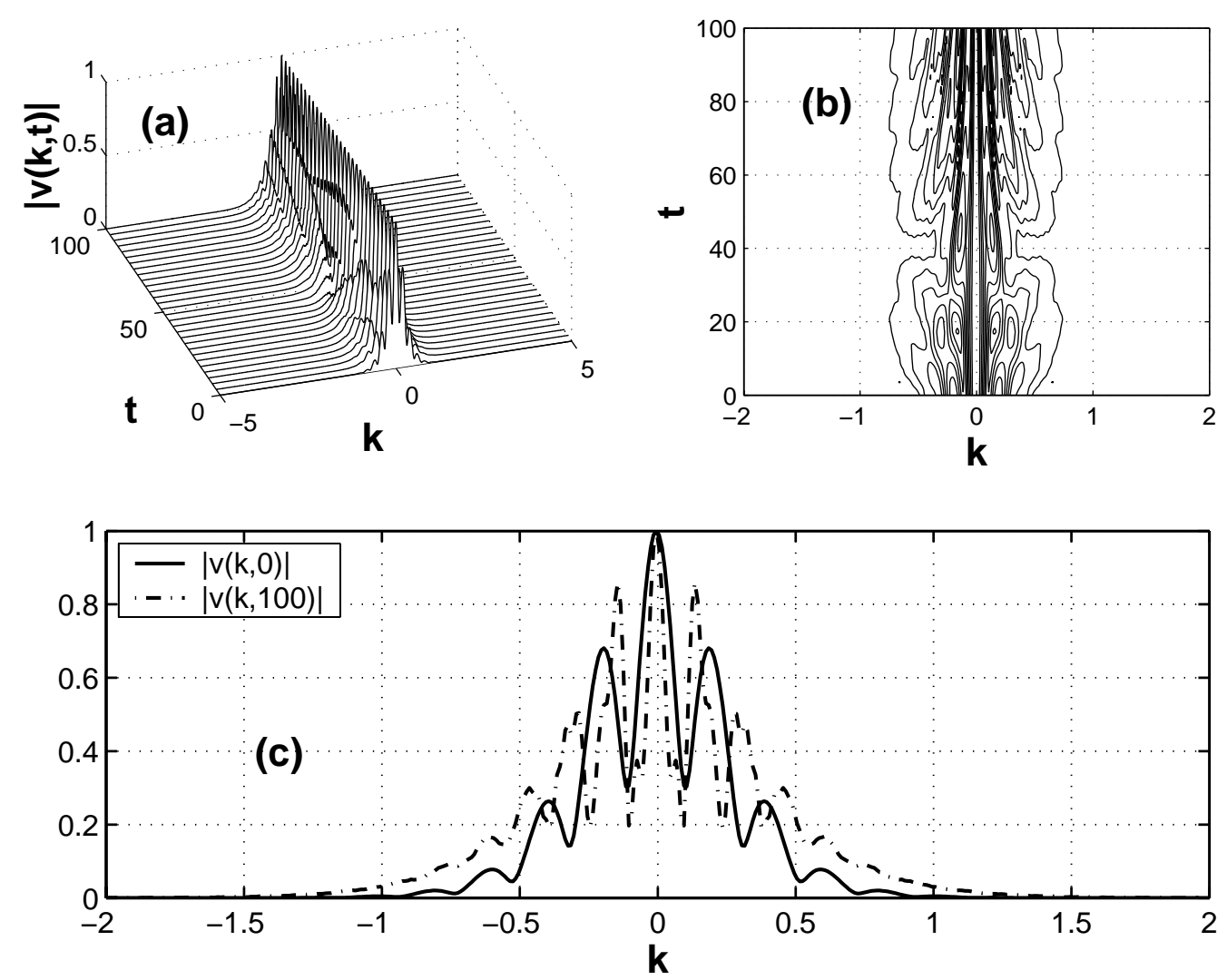

Fig. 5. (a) Evolution of the normalized Fourier transformed spectrum $|v(k, t)|$ as defined in (24). (b) The contour plot clearly shows the two waves interaction at $t \approx 40$. (c) Broadening of the Fourier spectrum is observed at $t=100$ in comparison with the initial profile at $t=0$ due to the presence of the oscillatory tail of the small solitary-wave.

local minimum about $t=20$ in Fig. $4 \mathrm{~b}$ indicates the moment at which the three solitary-waves exist simultaneously right before their interaction. However, the fact that at $t \approx 40$ the total energy returns to the initial value indicates that the interaction is exactly elastic even though the whole process is almost elastic. To understand the interaction in more detail, we plot in Fig. 5a the evolution of the Fourier transformed $v(x, t)$ defined as

$$
v(k, t)=\frac{1}{2 \pi} \int_{-\infty}^{\infty} v(x, t) \mathrm{e}^{i k x} \mathrm{~d} x .
$$

The contour plot in Fig. 5b portrays the broadening of the Fourier spectrum as a result of the decay of the higher amplitude solitary-wave and its subsequent interaction with other waves, where the width of the spectrum becomes minimum at $t \approx 40$, at which the interaction occurs. From Fig. 5c, we also find the broadening of spectrum at $t=100$ in comparison with that at $t=0$, due to the coexistence of the three solitary-waves as shown in Fig. 4c.

Figure 6 shows the simulation for $B_{0}=3 A_{0}$ by using the same coefficients as in Figure 4. In Fig. 6a, we observe the decay of the higher amplitude solitary-wave, however, contrary to Fig. 4, the subsequent interaction of the three solitary-waves results in a bounded state of two solitary-waves, i.e. a 'bion', which travels together as a single coherent structure with a fixed peak-to-peak separation $[14,15]$. As for the case of Fig. 4, the interaction occurs at $t \approx 20$, as shown in Fig 6b, at which the energy loss is minimum but the total energy slightly fluctuates thereafter. The bion indeed demonstrates a remarkable robustness by keeping its shape even up to $t=10,000$. We have run many simulations with different sets of coefficients to conclude that the formation of a bion is a generic feature for the almost elastic interaction of the solitary-wave solutions of which the coefficients belong to the regions (I) and (III), in particular 

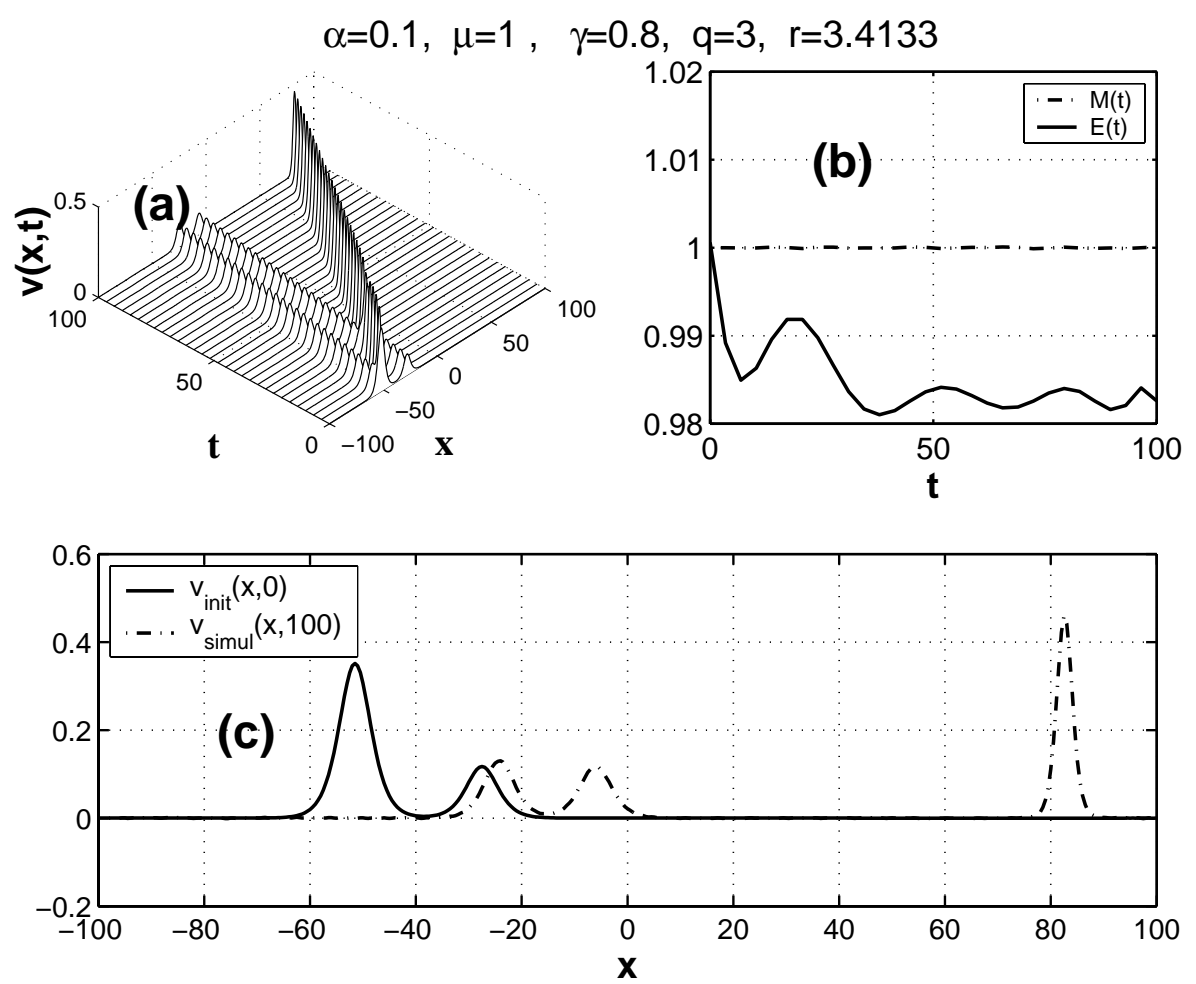

Fig. 6. (a) Evolution of two initial solitary-waves with amplitudes $B_{0}=3 A_{0}, A_{0}=0.117$, and $\delta=3$. The higher amplitude wave decays and subsequently interacts with the other wave on the right. (b) Almost elastic interaction occurs at $t \approx 20$, after which the bounded state of two solitary-waves, i.e. a bion, is formed, which shows stable propagation. (c) The initial vs. propagated profiles at $t=100$. A stable and fast traveling solitary-wave is shown at the rightmost (dash-dotted curve), while the bion does not travel.

for large nonlinear $r$ values, as in Figure 1d. The bion is an interesting feature, which has not been found in the previous numerical investigations [15-17] of the fifth-order $\mathrm{KdV}$ equations at various coefficient limits of (1), and it deserves more studies.

We also point out that the analytic solitary-wave solutions in Eq. (16) behaves much like the KdV soliton, which may shed light on the possibility of finding analytic N-soliton solutions.

\section{Conclusions}

We have found new analytic solitary-wave solutions, which satisfy the zero background condition at infinity, of the general fifth-order KdV equation under the balance constraint in (15) with the allowed coefficient regions in Fig. 1d, and discussed the method for obtaining kink-type solitary-waves and rational solutions with nonzero background [10-12]. By performing numerical simulations, we have shown the stability of bright solitary-wave solutions, of which the coefficients belong to the constraint region (I) in Figure 1d. Both the dark and bright solitary-wave solutions belong to the coefficient regions (II) and (III), respectively, and have been found to be stable. The question whether the stable solitary-wave solutions can exhibit soliton-like dynamics has been investigated, for example, with $\alpha$ and $\gamma$ which belong to the coefficient region (I) in Fig. 1d but with large nonlinear $r$ values, by considering the interactions of solitary-waves with two different amplitudes. We have concluded that the soliton-like interaction occurs due to the almost energy conserving elastic interaction as depicted in Figure 4b. Furthermore, in Fig. 6, it was shown that the bion can be formed as a result of almost elastic interaction nature of the two solitary-waves. In conclusion, we demonstrated for some coefficient regions in Fig. 1d the stability of the solitary-wave solution with zero background and the soliton-like behavior during the interaction of the waves. However, more extensive 
numerical simulations are needed to explore other coefficient regions too. Also, the mathematical question whether (1) can be transformed to a KdV hierarchy deserves future investigation.

[1] S. Kichenassamy, Nonlinearity 10, 133 (1997).

[2] S. Kichenassamy and P. J. Olver, SIAM J. Math. Anal. 23, 1141 (1992).

[3] T. B. Benjamin, J. L. Bona, and D. K. Bose, Phil. Trans. Roy. Soc. A331, 195 (1990).

[4] M. Weinstein, Commun. PDE 12, 1133 (1987).

[5] C. J. Amick and J. F. Toland, Euro. J. Appl. Math. 3, 97 (1992).

[6] B. Tian and Y. T. Gao, Z. Naturforsch. 51a, 171 (1996).

[7] J. Weiss, M. Tabor, and G. Carnevale, J. Math. Phys. 24, 522 (1983).

[8] B. Tian and Y. T. Gao, Phys. Lett A 209, 297 (1995).

[9] B. Tian and Y. T. Gao, Phys. Lett A 212, 247 (1996).

\section{Acknowledgement}

This research was supported by the Catholic University of Daegu in 2004.

[10] W.P. Hong and Y. D. Jung, Z. Naturforsch. 54a, 272 (1999).

[11] W. P. Hong, Nuovo Cim. B 114, 845 (1999).

[12] M. W. Coffey, Phys. Lett. A 295, 35 (2002).

[13] L. N. Trefethen, Spectral Method in Matlab (Siam, 2000).

[14] R. H. J. Grimshaw and B. A. Malomed, J. Phys. A 26, 4087 (1993).

[15] J. P. Boyd, J. Comp. Phys. 124, 55 (1996).

[16] C. I. Christov and M. G. Velarde, Physica D 86, 323 (1995).

[17] J. M. Hyman and P. Rosenau, Physica D 123, 502 (1998). 